\title{
OPTE special issue on technical operations research (TOR)
}

\author{
Armin Fügenschuh ${ }^{1} \cdot$ Ulf Lorenz ${ }^{2} \cdot$ Peter F. Pelz ${ }^{3}$
}

Received: 27 April 2021 / Revised: 1 May 2021 / Accepted: 1 May 2021 /

Published online: 12 May 2021

(c) The Author(s) 2021

\section{Aim and scope of the special issue}

Technical Operations Research is a bridging discipline that combines elements from engineering sciences (e.g., mechanical, electrical, civil, material sciences), computer science, mathematics and business economics. The aim of the presented special issue is to foster exact optimization in the engineering sciences. A role model is the classic Operations Research which has successfully brought discrete mathematics, combinatorial optimization and other formal disciplines to business engineering and management science. Therefore, we present a variety of contributions that focus on all stages of an engineering design process to derive sustainable systems designs that follow the statement, cf. (Pelz et al. 2021, Section 1.6):

"Maximize quality, subject to functionality!"

In general, an engineering design process consists of four major steps:

(i) the formulation of the desired task and quality,

(ii) defining the design space,

(iii) deriving the composition, and

(iv) the evaluation of realized task, quality and acceptance.

In phase (i) the needs result in a specified task. The expected quality dimensions regarding effort, availability, and acceptability are determined. In the subsequent phase (ii) the design space is formed out of the available resources, i.e., materials,

Armin Fügenschuh

fuegenschuh@b-tu.de

Ulf Lorenz

ulf.lorenz@uni-siegen.de

Peter F. Pelz

Peter.Pelz@fst.tu-darmstadt.de

1 Brandenburg University of Technology, Cottbus-Senftenberg, Germany

2 University Siegen, Siegen, Germany

3 Technical University Darmstadt, Darmstadt, Germany 
components, technologies and bounded by technical requirements. In phase (iii) the overall task is divided into sub-tasks forming a task structure. Sometimes, each subtask or a group of sub-tasks is assigned to one cyber-physical component fulfilling this sub-task. Then, by this assignment process a cyber-physical system is composed. In the final phase (iv), the system's task, quality and acceptance is verified and validated by means of evaluation metrics and methods.

For an in-detail evaluation of the given design space, algorithm-driven, mathematical optimization approaches are favoured, since as humans we are limited to combinatorial decision trees with only, say, $2^{2}$ to $2^{3}$ leaves, while machines and algorithms are able to handle much larger decision trees.

Afterwards, the applicability of these algorithmically derived system designs could then be validated by models with a higher fidelity or experiments. Therefore, Technical Operations Research (TOR) focuses on technical systems and the control and management of their processes. One example is the combination of individual modules into a larger technical system in the best possible way, when technical constraints as well as economic considerations have to be considered. In this way, TOR is meant to bring OR to new areas of applications, which implies the development of new models and algorithmic methods to solve them to proven global optimality. As a side effect, it stimulates the communication between the different areas of expertise, in particular, between engineers, mathematicians, computer scientists, and economists.

\section{Major topics of interests}

The "ideal submission" we had in mind when issuing the call-for-papers would show a mixture of the three core TOR disciplines:

- Problems, models and algorithms: We aimed at engineering problems that lead to new TOR research questions, formulated as mathematical optimization problems (e.g., (non-) linear, mixed-integer, ODE/PDE-constrained, robust, stochastic, bilevel, etc.), giving rise to the development of new or modified algorithmic approaches for a successful and scalable solution.

- Validation and verification: Typically, TOR algorithms use a coarse approximation of physical and technical constraints. Hence their solutions need to be validated and verified by, e.g., simulation models that carry a more refined description of the physics, or by real-world demonstrators and prototypes. This in return shows model-weaknesses and -limits, and can be used to improve formulations and refine models.

- Tools for engineers: A major idea of TOR is that the optimization tools do not replace engineers but extend their capabilities. The goal is to build tools such that the engineers can describe and model a problem at hand, and solve them supported by algorithms. This necessitates the development of tailored modeling languages for describing problem instances as well as interfaces to navigate and explore the solution space in a user-friendly manner. 
Following our call, we received several submissions, from which eleven were selected for publication. In accordance with this holistic system design approach, this selection fits particularly well into the scope of Technical Operations Research. A multitude of technical systems, algorithmic approaches, and tools for engineers are shown. This underlines the wide applicability of the afore mentioned ideas. In the following, we give a brief outline of the individual contributions to the broader field.

\section{Contributions}

An overview about different solution approaches that incorporate engineering knowledge in the solution process of modern mathematical optimization methods is shown by Weber et al. (2020) for fluid and thermofluidic systems. They explicitly focus on the applicability of derived quantitative approaches in the engineering domain and present exemplified software implementations and results. Multiple extensions of a given basic model are shown to derive more resilient systems through the usage of quantified programming, the integration of heat transfer and dynamic behavior. It is supplemented by detailed algorithmic approaches to increase the solution speed of a developed branch-and-bound with engineering specific knowledge and further algorithmic improvements.

The application of mathematical optimization methods for water supply system design and operation provides the capacity to increase the energy efficiency and to lower the investment costs considerably. A system approach for the optimal design and operation of pumping systems in real-world high-rise buildings, is presented in Müller et al. (2021). Here, different booster station topologies, i.e. parallel and series-parallel central booster stations as well as decentral booster stations, are considered. To confirm the validity of the underlying optimization models with realworld system behavior, validation results based on experiments conducted on a modularly constructed pumping test rig are presented, as well. On the more mathematical side, the authors consider layout and control decisions for different load scenarios, leading to a Deterministic Equivalent of a two-stage stochastic optimization program. They use a piecewise linearization as well as a piecewise relaxation of the pumps' characteristics to derive mixed-integer linear models. Besides the solution with off-the-shelf solvers, they present a problem specific exact solving algorithm to improve the computation time. The performance and applicability of the solution approaches for real buildings are discussed and the technical aspects of the solutions from an engineer's point of view are analyzed, keeping in mind the economically important trade-off between investment and operation costs. The paper nicely shows the bandwidth of Technical Operations Research.

Next to water distribution systems we also present related approaches for the design and operation of real-world gas or energy networks. In Hennings et al. (2020), the authors explicitly consider a detailed modeling of compressor stations, which contain a high number of configurable components in a gas network and represent intersections of major transportation pipelines. They present a mixed-integer system model that considers compressor stations in more detail. The developed 
algorithms were tested based on real-world data and show a suitable solution time for a later real-world usage.

A second contribution in this special issue in the domain of gas network design and control is given by Hoppmann-Baum et al. (2021). They present parts of a decision support system named KOMPASS for the usage by dispatchers which control the system behavior. Additionally, they present algorithmic approaches to solve the underlying optimization model.

Krug et al. (2020) optimize district heating networks using a complementary-constrained nonlinear optimization model, whose solution yields a time-dependent control of the network. The technical constraints here are related to the physics of the mixing of water with different temperatures. This leads to nonlinear partial differential equations, which are then discretized in time and space. Using this approach, they are able to solve instances having a practically relevant size.

Hollermann et al. (2020) are concerned with the design of sustainable energy systems, balancing economical, environmental, and social needs. They formulate a two-stage decision problem, where the first stage deals with the actual design of the network, and the second stage with its operation. Parameter uncertainty is additionally taken into account. They then compute the Pareto front, which shows the trade off between economical and environmental goals. They derive a mixed-integer programming formulation and demonstrate that a standard numerical solver is able to solve real-world instances.

Reintjes and Lorenz (2020) develop two mixed-integer programs for the design of truss topologies. The model choice depends on the respective design space: The first of the two can be used for powder-based additive manufacturing, whereas the second is for support-free lattice structures. Technical constraints are enforcing the mechanical stability of a generated solution. They embed the solution algorithm into a computer aided design tool, so that the design engineer has an easy access to their method. Their contribution is a prime example for Technical Operations Research, since it involves all three core TOR disciplines mentioned above.

Hofmann and Wenzel (2020) study industrial robot systems in manufacturing. Since robots perform repetitive tasks, this leads to the analysis of cycles and their minimum completion time. The authors demonstrate how to apply periodic event scheduling models to this, so that an optimal and conflict-free movement for a robot can be determined. Their method is applied to a real-world robot system. Another manufacturing problem with technical real-world constraints is picked up in Bähr et al. (2021). The authors consider two mathematical problems that occur in the layer-wise production process of a work-piece using wire-arc additive manufacturing. As the first task, an automatic construction of a honeycomb structure is performed, given the boundary of a shape of interest. In doing this, the authors employ Lloyd's algorithm in two different realizations. For computing the incorporated Voronoi tesselation they consider the use of a Delaunay triangulation or alternatively, the eikonal equation. These approaches are compared and modified with the aim of combining their respective advantages. Then in the second task, to find an optimal tool path guaranteeing minimal production time and high quality of the work-piece, a mixed-integer linear programming problem is derived. The model takes thermal conduction and radiation during the process into account and aims 
to minimize temperature gradients inside the material. Its solvability for standard mixed-integer solvers is demonstrated on several test-instances.

Zazai and Fügenschuh (2021) demonstrates a further branch of variety for Technical Operations Research. Planning the construction of new transport routes or power lines on terrain is usually carried out manually by engineers, with no guarantee of optimality. Here, the authors introduce a new approach for the computation of an optimal trajectory for the construction of new transit routes and power lines between two locations on a submanifold $U \subset \mathbb{R}$ representing the topography of a terrain. $U$ is approximately modeled by a special weighted grid. On this grid, the shortest paths for the construction of new routes are determined, whereby several optimization criteria are considered. A shortest path algorithm is used to compute optimal compromises for the construction of new routes.

The assessment of the ecological impact of different powertrain concepts is the topic of Esser et al. (2021). This topic is of increasing relevance considering the enormous efforts necessary to limit the global warming effect due to the man-made climate change. This contribution lives from its relevance for Society and impressively shows the long path from intuitively capturing relevant aspects of reality down to the formulation of abstract models. Within this contribution, the authors adopt existing methods for the optimization of electric and hybrid electric powertrains using a vehicle simulation environment and derive a method to identify the ecological potential of different powertrain concepts for a set of technological parameters in the reference year 2030. By optimizing the parametrization for each powertrain concept and by adapting the respective operating behavior specifically to minimize the ecological impact, a reliable and unbiased comparison is enabled.

\section{Conclusion, outlook and acknowledgement}

The content of the selected reviewed papers shows the power of exact optimization methods and TOR in general.

For the further development of the TOR methodology, the thesis is to be established that every engineering design process can in principle be mapped to a constrained optimization problem, provided that the function and quality of the technoeconomic system is described consistently and with low uncertainty and the design space is described completely. The TOR methodology thus has two sides of the same coin, on the one hand the modeling of constraints and objectives and the associated metrics, and on the other hand the development of increasingly user-friendly, robust and faster frameworks that use solvers such as a MINLP solver.

We would like to thank Philipp Leise who supported the three of us in ensuring the quality of a valuable special issue. We would like to thank all the authors of this special issue. We hope you enjoy the special issue in the same way we do it.

Armin Fügenschuh, Ulf Lorenz, Peter F. Pelz

Funding Open Access funding enabled and organized by Projekt DEAL. 
Open Access This article is licensed under a Creative Commons Attribution 4.0 International License, which permits use, sharing, adaptation, distribution and reproduction in any medium or format, as long as you give appropriate credit to the original author(s) and the source, provide a link to the Creative Commons licence, and indicate if changes were made. The images or other third party material in this article are included in the article's Creative Commons licence, unless indicated otherwise in a credit line to the material. If material is not included in the article's Creative Commons licence and your intended use is not permitted by statutory regulation or exceeds the permitted use, you will need to obtain permission directly from the copyright holder. To view a copy of this licence, visit http://creativecommons.org/licen ses/by/4.0/.

\section{References}

Bähr M, Buhl J, Radow G, Schmidt J, Bambach M, Breuß M, Fügenschuh A (2021) Stable honeycomb structures and temperature based trajectory optimization for wire-arc additive manufacturing. Optimization Eng. https://doi.org/10.1007/s11081-020-09552-5

Esser A, Eichenlaub T, Schleiffer JE, Jardin P, Rinderknecht S (2021) Comparative evaluation of powertrain concepts through an eco-impact optimization framework with real driving data. Optimization Eng. https://doi.org/10.1007/s11081-020-09539-2

Hennings F, Anderson L, Hoppmann-Baum K, Turner M, Koch T (2020) Controlling transient gas flow in real-world pipeline intersection areas. Optimization Eng. https://doi.org/10.1007/ s11081-020-09559-y

Hofmann T, Wenzel D (2020) How to minimize cycle times of robot manufacturing system. Optimization Eng. https://doi.org/10.1007/s11081-020-09531-w

Hollermann DE, Goerigk M, Hoffrogge DF, Hennen M, Bardow A (2020) Flexible here-and-now decisions for two-stage multi-objective optimization: method and application to energy system design selectio. Optimization Eng. https://doi.org/10.1007/s11081-020-09530-x

Hoppmann-Baum K, Hennings F, Lenz R, Gotzes U, Heinecke N, Spreckelsen K, Koch T (2021) Optimal operation of transient gas transport networks. Optimization Eng. https://doi.org/10.1007/ s11081-020-09584-X

Krug R, Mehrmann V, Schmidt M (2020) Nonlinear optimization of district heating network. Optimization Eng. https://doi.org/10.1007/s11081-020-09549-0

Müller TM, Leise P, Lorenz IS, Altherr LC, Pelz PF (2021) Optimization and validation of pumping system design and operation for water supply in high-rise buildings. Optimization Eng. https://doi.org/ 10.1007/s11081-020-09553-4

Pelz PF, Groche P, Pfetsch M, Schäffner M (2021) Uncertainty in Mechanical Engineering. Springer, NewYork

Reintjes C, Lorenz U (2020) Bridging mixed integer linear programming for truss topology optimization and additive manufacturing. Optimization Eng. https://doi.org/10.1007/s11081-020-09541-8

Weber JB, Hartisch M, Herbst AD, Lorenz U (2020) Towards an algorithmic synthesis of thermofluid systems. Optimization Eng. https://doi.org/10.1007/s11081-020-09564-1

Zazai MF, Fügenschuh A (2021) Computing the trajectories for the development of optimal routes. Optimization Eng. https://doi.org/10.1007/s11081-020-09569-w

Publisher's Note Springer Nature remains neutral with regard to jurisdictional claims in published maps and institutional affiliations. 